\title{
An improvement in the energetic efficiency of a building using daylight in the light control system
}

\author{
Stawomir Sowa ${ }^{1, *}$ \\ ${ }^{1}$ Institute of Electric Power Engineering, Faculty of Electrical Engineering Poznan University of \\ Technology, Poland
}

\begin{abstract}
Increasing the energy effectiveness of buildings focuses mainly on limiting the use of heat and electricity. The benefits from such activities have an impact on the cutting of running costs and assist in environmental protection thanks to the limited demand for primary energy. It is extremely important for the whole of humanity to perform actions to improve the energy effectiveness of buildings. One of the ways of limiting the use of energy for lighting, when it comes to electricity, is to implement a control system, which would enable limiting energy consumption, while giving proper user comfort and safety at the same time. This article describes the legal aspects connected with energy effectiveness and it also deals with a lighting control system that uses daylight.
\end{abstract}

\section{Efficient energy use}

The term "efficiency" is widely used in many areas of our lives. By definition, it is assumed that efficiency describes a degree and a level of implementation of previously established objectives. In a more detailed approach, efficiency is understood as economy, and in a wider perspective, as effectiveness and advantages. However, from the point of view of energy use, this definition of efficiency is insufficient. The Energy Efficiency Act defines efficient energy use as a ratio of the achieved effect size of a facility's technical device or system in typical conditions of their use or operation for energy consumption by that facility's technical device or system, or following a service performed to achieve that effect [1]. In the simplest way, it can therefore be said that efficient energy use is a ratio of the achieved result to the amount of energy used to achieve that effect. Of course, we strive to achieve the required effect with the lowest energy expenditure possible. The improvement in the efficient energy use will therefore mean actions taken to limit consumption of the energy needed to achieve the required effects, improve the performance of equipment and processes, and limit losses. In practice, taking actions to improve energy efficiency frequently means that certain financial expenditures are required. The economic aspect is one of the main reasons for taking actions to implement solutions, technologies, or equipment which ensure a reduction in energy consumption. The problem of financing

\footnotetext{
*Corresponding author: Slawomir@Sowa.poznan.pl
} 
actions aiming at a reduction in energy consumption is frequently considered in terms of the time needed to achieve a return on that investment. However, it should be remembered that the use of equipment or components leading to savings also usually results in an improvement in the comfort or safety of the use of rooms or facilities.

\subsection{Legal acts concerning efficient energy use}

\subsubsection{Directives and Communications of the European Union}

For many years, the European Parliament has been focusing on the energy efficiency of buildings. This subject was addressed both in Polish and European legislation. The aspect of improved energy use was considered in a document from 2006 [2], establishing a plan for using the energy efficiency potential and ensuring Europe remained one of the most effective regions of the world in terms of energy consumption. Currently, a valid legal act is the EU Directive [3] of 2012 on energy efficiency, which assumes a $20 \%$ reduction in primary energy by 2020 . This document also obliges the member states to introduce actions aimed at a reduction in final energy consumption by $1.5 \%$ per year. In the communication published in 2014 on energy efficiency [4], the Commission established that additional efforts of member states were needed, as it was visible in its analyses that by 2020 the EU would be able to achieve a reduction in energy consumption at a level of only about 18$19 \%$.

\subsubsection{Regulations and Acts in force in Poland}

In recent years, works were undertaken in Poland to adapt relevant standards and regulations to the European Union requirements concerning energy efficiency. The EU recommendations are fulfilled by the Act of 2011 [5], specifying mechanisms aimed at energy savings, and in particular, at its better use by the end consumer.

\subsection{Improvement in the energy efficiency of buildings}

The improvement of the energy efficiency of buildings focuses mainly on activities related to their use and concerns, in particular, heating, lighting, ventilation, and hot water consumption.

Heating, cooling and air conditioning will require a precise temperature control according to the needs of each room. This can be achieved by using simple control devices, e.g., a thermostat. A popular and important element influencing savings on the heating and cooling of a facility is the thermal insulation of walls, floors and roof, as well as the sealing of window frames. The use of energy efficient heating equipment and a heat recovery system to heat rooms, as well as the use of gravitational ventilation will contribute significantly to an improvement in energy efficiency.

Electricity is the second most important factor influencing the energy efficiency of a facility. The use of energy efficient devices and sources of light represents a fundamental step towards a reduction in electricity consumption. Lighting, which may represent even up to $20 \%$ of the total costs of a facility's energy consumption, should be installed in a way to ensuring the maximum use of lighting intensity. Another factor is the use of energy efficient sources of light, as mentioned earlier. The implementation of a control system which ensures that the lighting works with the required intensity wherever and whenever it is necessary, also contributes to a reduction in energy consumption. In the subsequent chapter 2 of this paper methods used to control lighting are discussed. On the basis of the 
conducted studies, distributions of light intensity in rooms were obtained (Fig. 1), helping to develop a lighting control system (Fig. 2).

\section{Methods for lighting control}

Currently, there are many systems available used to control lighting. In fact, the technical aspect itself has practically been solved. However, the problem concerns the way in which the lighting system can actually be controlled. The issue of control is of great importance with respect to energy efficiency, because without appropriate control, energy consumption using a lighting system does not depend on the availability of daylight. Algorithms for lighting control were developed, taking into account conducted studies of light intensity distribution, which use daylight in their control [6].

\subsection{Control of a switch on/off type}

This is a basic control method, in which lights are switched off when the daylight intensity at a workplace or a location occupied by users reaches the sufficient required level, e.g., $500 \mathrm{~lx}$. Of course, in that case the presence of a user should also be taken into account. The most common and simplest solution is installation of a PIR-based motion detector. It is important to ensure an adequate delay for switch on and switch off parameters, to prevent the undesirable flickering of the light. The conducted studies and calculations showed that this method of lighting control may lead to savings in energy consumption of up to ca. $45 \%$ [7].

\subsection{Control of a dim/brighten type}

This is a second way for controlling the lighting system, in which additional artificial lighting can be provided at places where the light intensity is too low. The amount of energy to be used to ensure that additional lighting reaches a required level of lighting intensity in a given room has been determined in relevant studies. The calculations showed that this method of lighting control may limit energy consumption by ca. $48 \%$ [5]. It should be remembered that savings at this level are possible when a group or a row of lighting fixtures is controlled independently. A control of the dim/brighten type performs particularly well in deep rooms, where the required spot lighting intensity can be ensured by adding artificial light. 


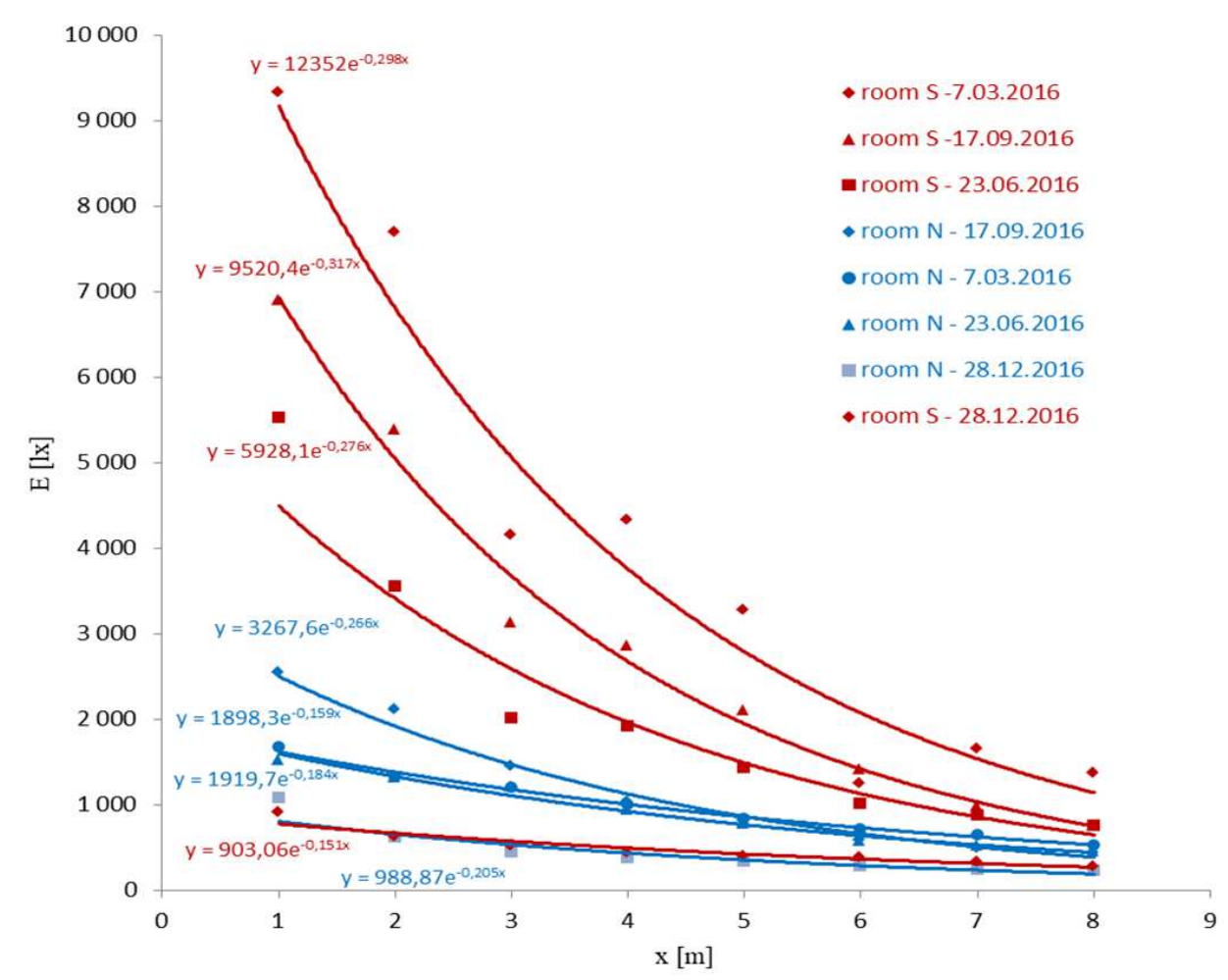

Fig. 1. Light intensity distributions in rooms on the $\mathrm{N}$ and on $\mathrm{S}$ side.

\subsection{Control of a dim/brighten type using daylight}

This is the most advanced effective control of lighting, resulting in the largest savings in energy consumption. In this method of control, an additional sensor is used to measure light intensity and decides whether the lighting fixtures in a given location should be switched on, and at what intensity. The most effective way would be to install light intensity sensors along the full width of the room, informing on the acceptable or sufficient light intensity, as required in a given location. This solution is very problematic, as it would require the installation of a row of sensors at a reference level, and this would hinder the free use and reliable measurement of the intensity level. The conducted studies on the light intensity distribution in rooms allowed specifying the conditions in which individual lighting fixtures should be switched on and brightened, or switched off and dimmed. An example of light intensity distribution characteristics in rooms is shown in Fig. 1. Measurements were conducted in rooms located on the $\mathrm{N}$ and on $\mathrm{S}$ side. The chart shows the dependence of luminance $\mathrm{E}$ from distance $\mathrm{x}$, which is measured from window to the opposite wall. The chart presents the distribution of luminance in a room, measured from the South (S) - red line. The blue lines, in turn, indicate the luminance measured from the North $(\mathrm{N})$. Values of the light intensity, at which adjustments of the artificial lighting are required, can be read from the curves. As visible in the charts presented in Fig. 1, the performed measurements of the light intensity distribution also showed that the light intensity distribution in rooms is not influenced by their orientation. The measurements showed that, obviously, in this case the environment in which the facilities are located and the furnishing in rooms are of importance. 
A control of this type can be installed using numerous available automatic control systems. A diagram in Fig. 2 presents the connections of components controlling the lighting system using daylight, implemented in the KNX system. With this standard, it is possible to program components of the system to switch on/off and dim or brighten individual rows of lighting fixtures, taking into account the preset values of daylight intensity outside of the facilities. In this system, the use on an internal light intensity sensor was also proposed, additionally verifying the indoor light intensity. Unexpected obstacles, dirty windows or the accidental covering of the external sensor, $1 x_{0}$, will result in verification of the indoor light intensity by the sensor $1 \mathrm{x}_{\mathrm{i}}$.

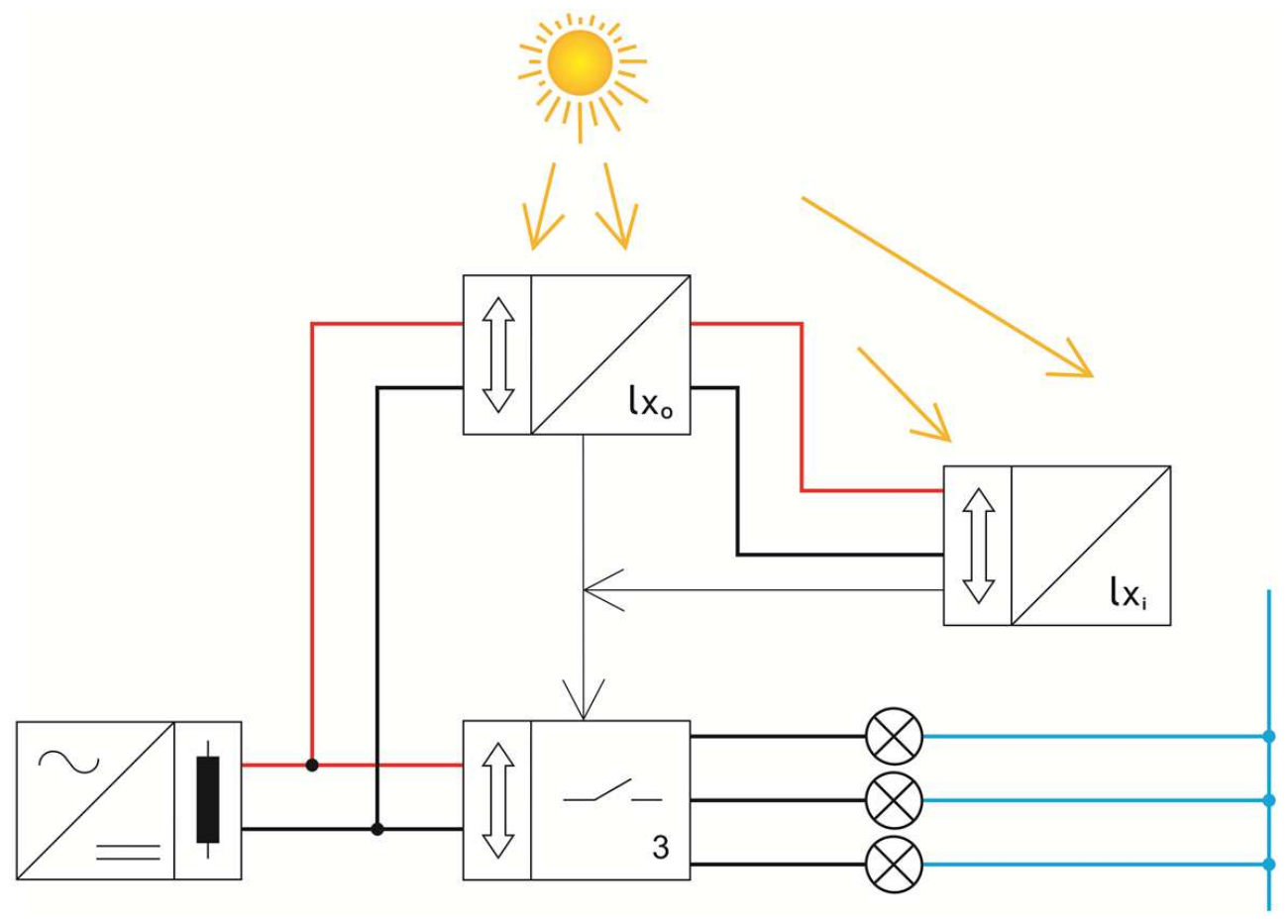

Fig. 2. Simplified diagram of lighting control in the KNX system.

\section{Savings resulting from lighting control}

The studies aimed to measure the light intensity distribution were conducted in rooms of a school in Swarzędz near Poznań. The measurements were conducted for 18 months in various weather conditions. During the measurements it was found that users did not turn the light off when leaving rooms. It was also observed that lighting switched on at the beginning of work was frequently left switched on for the whole day, even though enough daylight reached that room. The most effective way to eliminate these situations and achieve savings is to implement an automatic system which controls the lighting. The preceding chapter 2 of this paper discusses typical systems used to control lighting. In the conducted studies on light intensity, the distribution values at which the lighting can be completely or partly switched off were determined. Additionally, the intensity of artificial lighting, needed to provide the required light intensity at a given location, can also be specified. On the basis of conducted studies, threshold values were determined for the light intensity at which artificial lighting can be switched off at relevant locations. It was established that when the daylight intensity outdoors was at a level of $12000 \mathrm{~lx}$, the 
required value of light intensity, i.e., $500 \mathrm{~lx}$, was ensured in the whole room. When the light intensity outdoors dropped to $9000 \mathrm{~lx}$, then one row of lighting fixtures on a side opposite to the windows had to be switched on. This situation continued until the daylight intensity decreased to $70001 \mathrm{x}$. A drop below that value resulted in a need to switch the second, middle row of lighting fixtures on. The studies found that when the daylight intensity outside was below $5000 \mathrm{~lx}$, then the third row of lighting fixtures had to be switched on. In this situation, the whole lighting system in the room must be switched on, as the lighting intensity is insufficient along the whole width of the room. The use of a control system to switch the lighting off when a room is unoccupied and to switch relevant rows of artificial lighting off at a relevant daylight intensity will result in significant savings in energy consumption by the lighting system. Using the results of the light intensity distribution analyses in the room, relevant calculations were performed and it was estimated that the savings may reach up to $47 \%$ of total energy consumption by the lighting system, depending on the control system implemented. The calculations took into account the average time of a user's absence in rooms and daylight intensity values at which relevant rows of lighting fixtures could be switched off.

The current studies focus on the use of light intensity sensors installed in rooms which will verify light intensity in rooms regardless of the information provided by outdoor sensors, while taking into account temporary obstacles such as elements of furnishing or users present in a room. In this respect, the most important task is to determine an optimal location for these sensors to prevent false results which will lead to ineffective control of lighting in the room.

\section{Conclusion}

The improvement of the energy efficiency of facilities is reflected in directives, standards and regulations in Poland, Europe and all over the world. Mankind should be interested in reducing energy consumption, which would lead to a reduction in its production and to the lower consumption of primary energy, including conventional energy. A reduction in the consumption of fuels, such as fossil fuels, would have a significant environmental impact. One of options for limiting energy consumption for lighting purposes is to ensure the maximum use of natural light. This can be achieved, for example, by installing an automatic lighting control system that will provide the required light intensity in the workplace or a location occupied by a user. The use of the control system reduces electric power consumption by the lighting system at locations and times when its use is not required (user's absence) or when the required daylight intensity is ensured. The conducted studies and calculations show that with these improvements, savings in electric energy consumption can reach 45-47\% [6]. Additionally, the use of energy efficient sources of light will increase these savings even further, thus improving the energy efficiency of buildings.

\section{References}

1. Energetic Efficiency Act, art. 2, pkt. 3, Dziennik Ustaw RP, 20.05.2016r., Warszawa.

2. Communication from the Commission - Action Plan for Energy Efficiency: Realising the Potential, 19.10.2006, Brussels.

3. Directive 2012/27/EU of the European Parliament and of the Council of 25 October 2012 on energy efficiency 2012/27/UE, 25.10.2012, Brussels.

4. Communication from the Commission to the European Parliament and the Council, 23.07.2014, Brussels. 
5. Energetic Efficiency Act, 15.04.2011, Dziennik Ustaw Nr 94, Poz. 551

6. Sowa S., Gielniak J. Lighting control algorithms in a school venue using KNX system. Electrical Engineering No 96, s. 153-162, Poznań 2018

7. Sowa S., Kamińska A., 2018. Predicted reduction of energy consumption in the school building by lighting control with KNX system. Przeglad Elektrotechniczny, 2/2018, $193-197$. 